\title{
Nostalgia as an emerging property of the Projective Consciousness Model
}

\author{
Manik Bhattacharjee ${ }^{* \dagger}$, David Rudrauf* ${ }^{* \dagger}$ \\ *Department of Psychology and Educational Sciences, University of Geneva, Geneva, Switzerland \\ ${ }^{\dagger}$ Swiss Center for Affective Science, Campus Biotech, University of Geneva, Geneva, Switzerland \\ ${ }^{\ddagger}$ Centre Universitaire d'Informatique, University of Geneva, Geneva, Switzerland
}

\begin{abstract}
The projective consciousness model (PCM) is a recently developed computational model of consciousness that allows an agent to project itself by imagination into an internal world model constantly updated through Bayesian inference from sensory evidence. The PCM acts to minimize free energy (FE) which is related to the divergence between predicted or preferred states and experienced states. The PCM uses its imagination to evaluate non-local situations. To increase psychological realism, we added a basic episodic memory to the PCM, which allows an agent to explicitly project itself in its past. As the PCM agent explores simulated worlds, it keeps a memory of its location and experienced FE across multiple layers of appraisal. When FE cannot be further minimized across possible local directions of action, the agent projects itself across space based on prior beliefs about either the present or the past, in an attempt to further lower its FE. Using simulations, we show that recalling pleasant memories allows a PCM agent to lower $\mathrm{FE}$, in situations where it is stuck with no other solution for $\mathrm{FE}$ minimization based on direct action or projections of possible future actions. Nostalgia is often defined as taking pleasure in recalling positive, soothing past events as a result of negative feelings in the present. The behavior of the PCM, which emerges from combining projective mechanisms, FE minimization and an episodic memory, is consistent with this definition.
\end{abstract}

Index Terms-nostalgia, projective consciousness model, episodic memory

\section{INTRODUCTION}

The Projective Consciousness Model (PCM) is a recently introduced computational model of consciousness [12], [13], [20]. It features a local point of view on an internal model of the world (i.e. the agent's knowledge about the current state of the world) put in perspective through projective geometry as a method of conscious access and framing of information integration. A given perspective on the world model defines the field of consciousness (FoC) of the agent at a given time. This is phenomenologically analogous to the so-called Cartesian theater of consciousness [4] but does not yield a problematic infinite regress (see [12]). The internal world model being accessed in perspective is constantly updated through active Bayesian inference and free energy (FE) minimization [7]. FE minimization is also used to select actions based on imaginary stances anticipating alternate situations. A PCM agent thus appraises the state of its representation of the world and the consequences of its actions through the projection of a series of fields of consciousness (FoC) and seeks FoCs that minimize FE in order to drive its actions, in a manner that functions as a method of global optimization. In the PCM, FE quantifies not only violation of expectations or surprise (defined as divergence between prior beliefs and sensory evidence), but also violations of personal preferences, along multiple dimensions of appraisal related to internal preferences, such as pleasure, safety or norm compatibility.

To avoid getting stuck in local minima of FE, the PCM implements an imagination engine that is a natural consequence of its reliance on projective geometry. It can project itself inside its internal world model as if it was moving through the world as modeled in its memory, searching for more optimal situations as a model of possible future states. The simulated situation selected through FE minimization is then used to direct a PCM agent towards a newly defined goal expected to lower its internal FE. This allows PCM agents to pass through less than optimal situations such as elevated danger in the hope of reaching a better state, with the expected overall decrease in FE acting as a motivational force.

A prominent framework in affective science and emotion theory is appraisal theory [10], [14]. It proposes that emotional reactions are driven by cognitive evaluations, or appraisals, about the personal relevance of situations (e.g., a situation appraised as dangerous will elicit avoidance behavior as part of a fear episode), notably regarding (in)compatibility with goals, desires, beliefs, and expectations [5]. Appraised goal compatibility leads to positive emotions, whereas incompatibility leads to negative emotions such as anxiety, fear, frustration, or anger. Appraisal theory generally subscribes to a componential definition of emotion [6]

encompassing experiential, cognitive, motivational, physiological, and behavioral components. In PCM terms, experiential component is akin to experienced FE level and derivatives; estimated FE levels for possible actions in relation to current FE act as a motivational force and directs the PCM to approach or avoidance behaviors.

Joffily and Coricelli proposed a first model to interpret FE dynamics as emotional processes [9], linking FE values with valence, such as a high FE being akin to a negative emotional valence, and a low free energy to a positive valence. They modeled a range of emotions as variations of experienced FE using first and second FE time-derivatives. For example, relief was described as a negative first and second FE derivative, meaning that FE is decreasing faster and faster. The approach was limited in scope and implied a narrower understanding of FE in a context difficult to generalize. The presence of multiple 
dimensions of appraisal and their relation to FE was not considered, nor the previously expected $\mathrm{FE}$ values in relation to the experienced value.

It is indeed essential to consider the problem of FE minimization as a multivariate problem, in which a multiplicity of motives and perspectives, possibly implying a quite contradictory set of goals and preferences, operate simultaneously and with complex conditional relationships. Moreover, in the PCM, specific components of FE related to appraisal layers are combined to yield an overall FE to minimize through a weighted sum that drives the dynamics of the agents' behavior as a function of perspective taking, with:

$$
F E=\sum_{i} F E_{i}
$$

The $w_{i}$ weights of the sum represent the importance given to each layer and can operate as a mechanism of emotion regulation aimed at adaptively tuning the agent sensitivity, as a trait or contextually. For example, being risk-adverse can be modeled by attributing a higher weight to the safety layer, so that small increases of FE related to the violation of the preference for safety are amplified in a manner that triggers avoidance behaviors above and beyond other contextual influences, e.g. low FE related to the satisfaction of hedonic pleasure, normally fostering approach behaviors. On the contrary, sensation seeking and risk-taking behaviors, e.g. the thrill of parachuting, can be obtained by lowering the weight of a safety layer, affording a stronger influence on hedonic layers in the FE minimization process. In this way, the diversity of people's reactions to an equivalent set of stimuli can be represented in a unified framework. This also allows to represent a larger set of emotions as clusters of FE layers' dynamics.

A key element in generating complex emotional states is the projective integration of FE dynamics as experienced in the past and in the present, and as expected both in the past and for the future. The dynamics of experienced FE are in our opinion insufficient to model the whole range of emotions. Relief for instance should be conceived in relation to both experienced (past and present) and expected (in the past and for the future) FE, such that having had a high expected FE causing anticipatory discomfort followed by an experienced FE lower than expected. This conceptualization of appraisal and emotion theory combining FE minimization, perspective taking, and projections at multiple time scales, across multiple appraisal dimensions is discussed in [13].

In the perspective of enhancing time consciousness in the PCM, we implemented a simple model of episodic autobiographical memory into the model. This allows PCM agents to project themselves in their own past. The goal of adding such a memory was to enable the PCM to learn from past experience, as it currently only considers the current state of the world, and to model the sense of the continuous self in a timeline in a manner that would be closer to human psychology.

Here, we explore the consequences of integrating such an episodic memory on the PCM emotional behavior. In partic- ular, we concentrate on a complex emotional state referred to as nostalgia.

Nostalgia is an emotion of particular interest in our context as it involves a projection into the past. It can be defined as a fond remembrance of positive past events that can be triggered by current discomforting states [16] or salient stimuli such as scent [11]. Recent theories of appraisal have emphasized the role of appraisal and reappraisal strategies in the context of emotion regulation in shaping resilience toward stressors (Kalisch et al., 2015; Sheppes et al., 2015; see also Gross, 1998). Reappraisal can be achieved by relating the current experience with previous events, which may be accessed through a nostalgic experience. Nostalgia is cross-cultural [8], but was historically mostly viewed negatively in psychology, or even as a pathological feeling. In recent years an increasing interest in the benefits of nostalgia offered a better characterization of this emotion. It has been shown to improve mood [2], [19], to improve the sense of self [1], [18] and to increase social bonding and emotional coping [15] through reappraisal.

We simulated a basic version of the PCM with an episodic memory and probed its behavior in relation to its past experiences. Does the process of FE minimization through perspective-taking yield solutions that find an optimum in evoking past states as part of the process of global optimization ?

\section{MATERIAL AND METHODS}

A simplified, 2D version of the PCM model [12] was reimplemented in Python: the PCM agent is moving on a 2D plane, and it has two FE appraisal layers corresponding to hedonic and safety appraisals. FE is an upper bound on surprise, that is $-\log (p(s \mid m))$, the negative logarithm of a probability density of an experienced sensory state $s$ under a given generative model of expectation $m$, which can relate to objective states of the world or prior preferences. More specifically, for a given experienced sensory state $s$ and an internal parameterization $\mu$ of the causes of that state $\Psi$ (e.g. contact with a pleasurable object), $F E(s, \mu)$ is equal to a term of energy, defined as the expectation, weighted by the variational density $q(\Psi \mid \mu)$, of the generative density $p(s, \Psi \mid m)$, minus the entropy of the variational density. In this contribution, we assumed the values of FE as being given by an internal computation that we did not explicitly model, and used arbitrary values between 0 , minimum FE, and 1, maximum FE, as our aim was to illustrate the role of projective mechanisms in FE minimization and not to consider the exact definition of FE or various ways to approximate it, which was beyond the scope of this contribution. The world itself was defined as a 2D, $200 \times 200$ matrix of pairs of values (one value for each appraisal layer) as a background and a few objects which have their own pair of values that may vary over time, all of them being related to arbitrarily, yet consistently defined value of $\mathrm{FE}$.

The main algorithm of the PCM was defined as follow:

- Perceptual inference: PCM agents look at the world ("World" in figures) using sensory evidence and update 


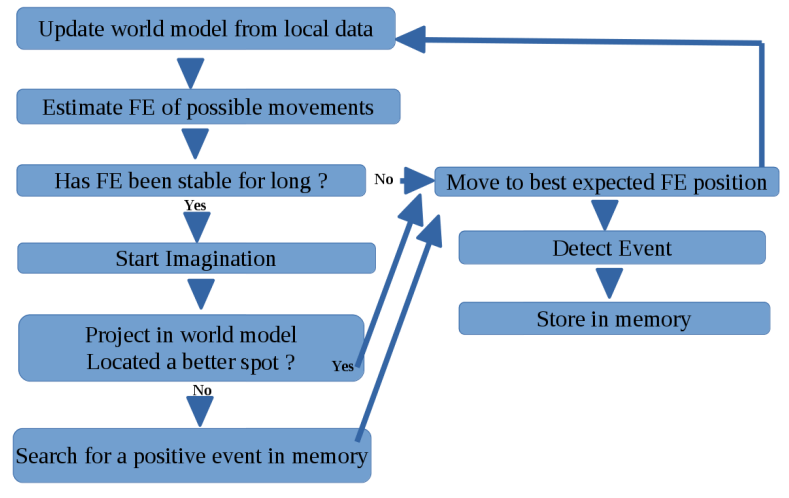

Fig. 1: Main algorithm.

their prior internal map of the world ("PCM World model" in the figures) accordingly;

- Local exploration of possible actions: PCM agents evaluate movements around their position according to the updated internal world model by computing expected FE for each action;

- Selection of action: PCM agents choose the optimal movement that should result in the lowest FE;

- Projective imagination: if the process of local optimization offers limited solutions of FE minimization, e.g. the world model is durably associated with similar values of FE in all directions, PCM agents use non-local or counterfactual projective imagination to seek better solution of FE minimization outside their immediate vicinity. This avoids the PCM getting trapped in local minima of FE by projecting itself in its world model and episodic memories.

For this last step, when the local gradient of FE is approaching zero and the PCM stays in a small zone for more than a time threshold, the PCM can be described as being in a state of bliss (very low, stable FE), in a state of despair (very high stable FE) or bored (average stable FE). Since PCM agents are driven by the goal of minimizing $\mathrm{FE}$, in the two latter cases in particular but also in the former case after a while, the imagination engine will switch on and the PCM will start projecting itself in space and time in search for a better solution.

We chose to trigger the imagination engine when the PCM stayed more than 40 time-steps in an 80 square pixels zone or more than 20 time-steps in a 10 square pixels zone. To prevent the PCM going out of the world space, the walls are defined to have very high FE values (100 times the maximum value of the environment's FE). This can be understood as the anticipation of an extremely dangerous state which must be avoided. The FoCs were simply approximated using a disk of 20 pixels diameter around the real or imaginary location of the PCM agent. Geometrical perspective was approximated by taking into account the distance of the pixels within a FoC from the location of the agent, and computing a weighted sum of the contribution of $\mathrm{FE}$ in the $\mathrm{FoC}$ and across layers of appraisal, with $\mathrm{FE}$ weights defined as the inverse of the pixel distance from the agent's location, following principles of perspective division. Each FoC was thus associated with an average FE representing the overall affective experience within it.

A simple model of episodic memory was added as follows. It was defined as a list of events where each event is a tuple of a mean location, mean time frame and a mean multi-layer FE. While the PCM algorithm is running, a new event is created when FE changes rapidly in a small time frame. To detect transitions, an arbitrary threshold was used on the absolute value of the second derivative of $\mathrm{FE}$, averaged using a sliding time window (width: 5 time-steps). Moreover, a minimum and maximum duration (10 to 200 time-steps) were defined for events in order to avoid inappropriately short or long events in the case of a rapidly changing FE or on the contrary very stable situations. The events were then indexed by time, mean location and by FE values (global as well as by appraisal layer).

When in imagination mode, PCM agents search their internal world model for a better non-local spot. To model sparse sampling of memory by the agents (versus a full exploration of it), ten random locations in the internal world model, were evaluated for expected FE levels each time spatial imagination was triggered. If a location was expected to be better because it had lower expected FE than the current location, it was chosen as a new goal by the agents. In such cases, when possible, agents were driven to travel towards the goal without regard for local FE, proportionally to the anticipated decrease in FE. If no better location could be found in the internal world model (corresponding to the expected current state of the world), PCM agents had the option of exploring their episodic memory and project themselves in their past, even for events and locations that would not be any more actually reachable, e.g. a past situation involving an object forever lost. In such situations, PCM agents looked up in the indexes of their episodic memory according to FE, trying to find events in the past that would improve their current situation. The probability of selecting an event $E_{i}$ from the list of events which have a $F E\left(E_{i}\right)$ lower than the current $\mathrm{FE}$ was defined as:

$$
p\left(E_{i}\right)=\frac{F E_{\text {current }}-F E\left(E_{i}\right)}{\sum_{j} F E_{\text {current }}-F E\left(E_{j}\right)}
$$

The randomly selected past event being better than the current state or any anticipated states, it would be considered in place of the other states to define the current FoC of the agent, as a reminiscent state. Following the general algorithm of the PCM, such reminiscent state is chosen as a new goal. If the event's location is still lower than the current FE in the internal world model, the PCM will move towards it. If the event is only reachable through the imagination because the current $\mathrm{FE}$ values at its location is now higher in the world model than at the time the event was stored, the PCM would evoke the event by imagination, projecting itself into a time and place associated with pleasant memories, but it would not actually move.

Practically, the currently experienced FE was computed as a mean of the present local FE and of the past FE at the 
goal site, thus overall minimizing FE among all possible other combination of states, e.g. present and future.

\section{RESULTS}

In the following simulation, the world was composed of a background with a linear gradient of FE in the hedonic layer with Gaussian noise changing at each time-step to simulate perception noise, and a constant FE in the safety layer with Gaussian noise. Four objects were present. Two were dangerous (red circles in figures) and the other two were pleasant (green circles in figures), with their pleasantness oscillating over time between slightly more and much more pleasant than the background. This was done in order to create a non-constant, changing world. Importantly, one of the pleasant objects was scheduled to disappear if the PCM would move near it and remain in its vicinity during 50 time-steps. The goal was to simulate an object that would become forever lost (e.g. the death of a loved one, or the loss of a valuable item).

The PCM started with an empty internal model of the world (prior beliefs about the world in which the agent spawns) where the expected FE values of the background were slightly lower than they would be in reality. This allowed the PCM to be curious about unexplored areas as they were expected to be more optimal than most already explored areas.

Figure 2 represents the simulation after a few time steps. The PCM agent moves in the world (a) following the local gradient of FE and updates its world model (b) from local data. The objects are shown as discs in the world, squares in the PCM memory. The maps and objects's FE values are shown using the green (low FE) to red (high FE) colormap, as a mean of the two appraisal layers. The path explored by the PCM is displayed as a black line on the world as well as on the PCM internal representation of the world.

In figure 3 the PCM has now reached a stable local optimum (blue arrow). After some time spent there, the spatial imagination engine switches on and finds a possibly better target location (red circle). The PCM thus starts moving towards it (red arrow). We can see in the world (a) that the location is not optimal, but it is better than the current one as estimated by the PCM from its internal world model (b). In (c) the FE level experienced by the PCM is plotted with black vertical lines showing the limit between two detected events, and the blue vertical line showing the spatial imagination switching on.

In figure 4 the PCM has reached a local optimum again and did not find a better spot using spatial imagination. It thus retrieved positive events from memory and used spatial imagination to project itself in one of the best memories. Comparing its memory to the current state of the event's location according to its world model, the PCM decides to move towards the previously encountered positive object (red circle and yellow arrow). The FE plot (c) displays the FE values experienced by the PCM, vertical black lines show the limit between two events. A blue vertical line is used to display the start of spatial imagination and a red vertical line for time imagination. The FE plot (c) in figure 4 shows the preceding stability in FE and the start of the movement which lowers $\mathrm{FE}$ as the expected low FE of the goal is combined with the local FE.

The memory map (d) represents the content of the episodic memory of the PCM. It shows a 2D map just as the world (a) and the PCM world model (b), but displays only a small circle located at the mean location of each recorded event. The color of the circle represents the mean FE experienced during the event, so pleasant events are displayed as light green dots, such as the one being recalled (red arrow) while unpleasant events are red. Mixed events are brown (e.g. the local optimum before time imagination - blue arrow).

Finally in figure 5 the PCM is in a similar situation, but the remembered event leads to a location which is currently not better than the current location. The remembered event (red arrow in (d)) is located in an area (red circle in (a) and (b)) which is not optimal anymore. The object that was the cause of the positive memory has disappeared and the PCM has updated its world model to remove it. The PCM does not move but evoking the memory lowers its experienced FE (red arrow).

The result of a hundred simulations is shown in figure 6 . The histogram shows the mean value of $\mathrm{FE}$ after local optimum search, after spatial imagination followed by local optimization, after time imagination followed by local optimization, and during mind-wandering, that is when the lost desired object can only be evoked mentally through imagination of the past. Error bars represent the standard deviation of the values.

This shows that the PCM using spatial imagination does not decreases its FE more efficiently than local optimization which was expected as the spatial imagination uses wrong priors on the world and do not provide accurately better targets than the current location in our simulations. When the PCM uses time imagination to go back to the place linked to a positive event (yellow bar), it allows it to reach a better location. As mind wandering takes place when the PCM has reached a point where it cannot find a better spatial projection and the past event evokes an unreachable object, it is already experiencing a low average FE. Remembering the event further reduces FE but not as much as reaching the location of an event that is still optimal.

\section{DISCUSSION}

The implementation of episodic memory and the ability to access it through imaginary projections in PCM agents were shown to improve the agents' ability to locate globally optimal places by guiding their focus on already well-known locations.

Nostalgia is often described as taking pleasure in recalling positive, soothing past events, as triggered by negative feelings. The observed behavior and associated emotions as understood within the PCM framework during the use of time imagination in relation to a lost object, were akin to these properties of nostalgia. The minimization of FE through imagination of the past functioned as a coping strategy in the 


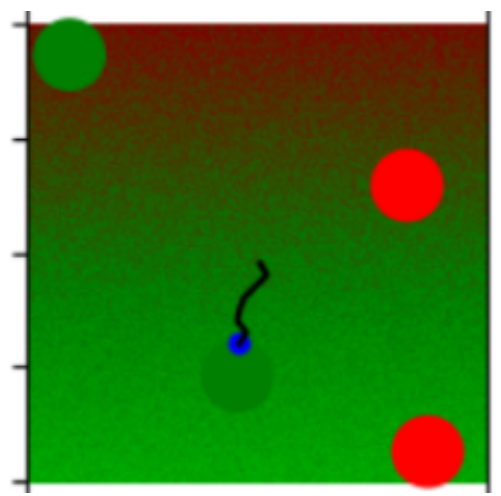

(a) World

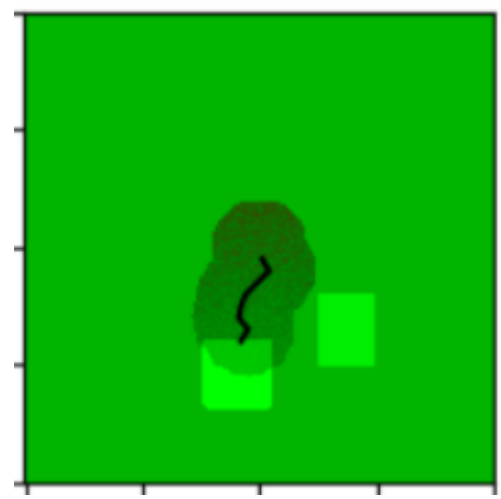

(b) PCM World Model

Fig. 2: The PCM is exploring the world. In black, the path it followed to its current position(blue dot).

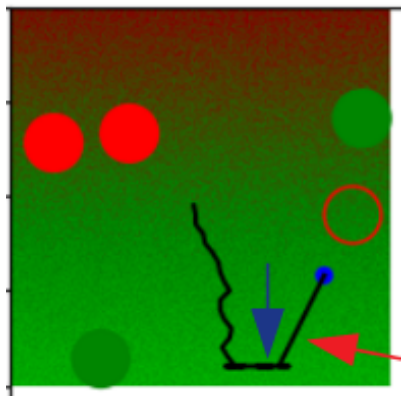

(a) World

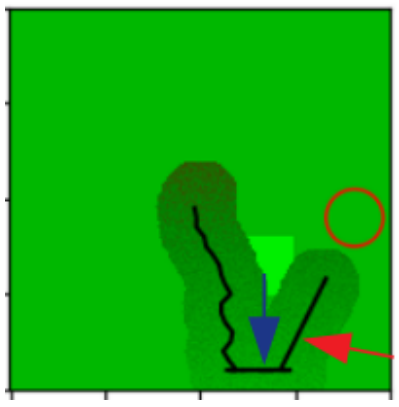

(b) PCM World Model

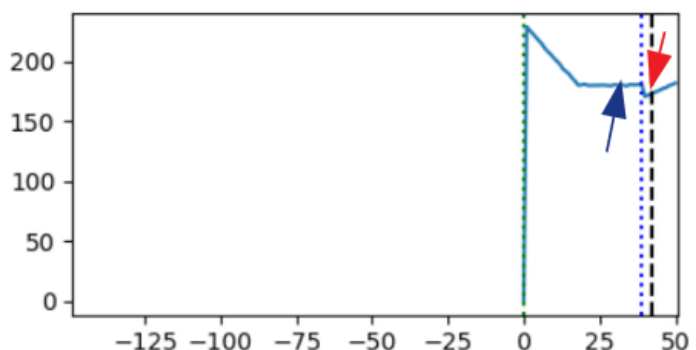

(c) Experienced FE timecourse

Fig. 3: After a stable time (blue arrow) spatial projection starts (blue vertical line in (c)) and finds a distant goal (red circle). The PCM moves towards it (red arrow).

face of difficult or non-optimal circumstances, and effectively acted as an improvement of the current mood of the agents.

Thus interestingly, even though the solution is not sustainable as it can only reside in an imaginary world, by evoking past comfort, e.g. successes, it offered a form of emotion regulation strategy. In more sophisticated implementations of PCM agents, and in real life, this might offer better conditions to tolerate the present and wait for the availability of better future solutions. Furthermore, because of the process of Bayesian updating intrinsic to the PCM, the effect on the present state of recalling better past events could soften the future memory of the present state and its reappraisal. This could relate to the positive bias humans have when retrieving old events [3].

It is interesting to note that nostalgia is not explicitly encoded into the model, but the behavioral, experiential and action tendencies of the componential aspects of this emotion are emerging from a combination of FE optimization and episodic memory access. This shows that it may not be necessary to explicitly encode emotions directly to create emotional agents, and that complex emotional states can be thought of as emergent properties of the complex internal dynamics of agents using memory, imaginary perspective taking and global optimization such as FE minimization over multiple dimensions of appraisal [13].

Even though the dynamics modeled here could account for central features of the concept of nostalgia, the implementation and simulations used for this article remain limited. Future work will have to model explicitly constructs such as that of self image in order to further account for documented effects of nostalgia and other emotional states [1], [17]. Nostalgia has also been shown to reinforce a sense of belonging and social bonding. Social relations were not simulated here, and thus the social benefits of nostalgia were absent from our simulations. Likewise, episodic memory recall was not influenced by the context apart from current FE values. Future implementations should make sure that recalled event choices may be influenced by contextual cues such as a similar location or a particular sensory input (e.g. scent-evoked nostalgia [11]) in order to get closer to experienced nostalgia in humans.

Moreover negative processes such as rumination are not accurately captured by the model here, but this could be related to the weight given here to spatial imagination as the primary imagination mechanism. In the case where spatial projection is ignored in favor of reexperiencing the past, behaviors akin to rumination will arise. Rumination on positive past events may arise from contextual or internal limitations in agents unable to envision better future states. 


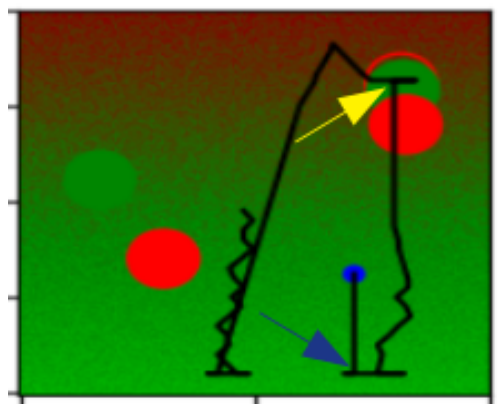

(a) World

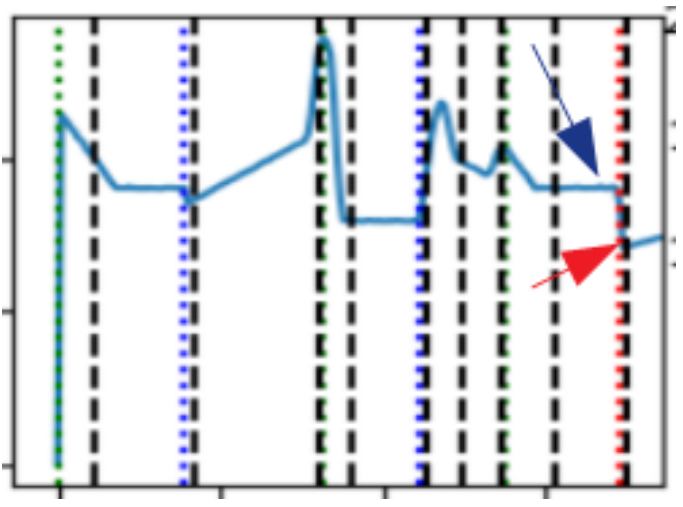

(c) Experienced FE timecourse

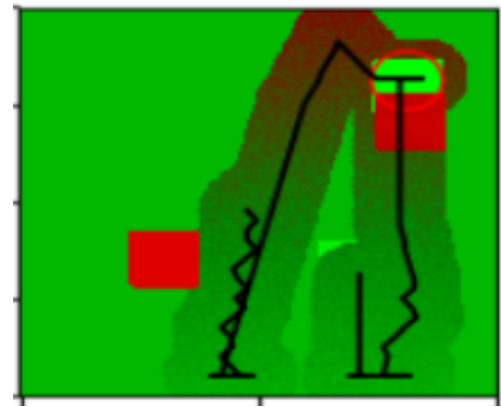

(b) PCM World Model

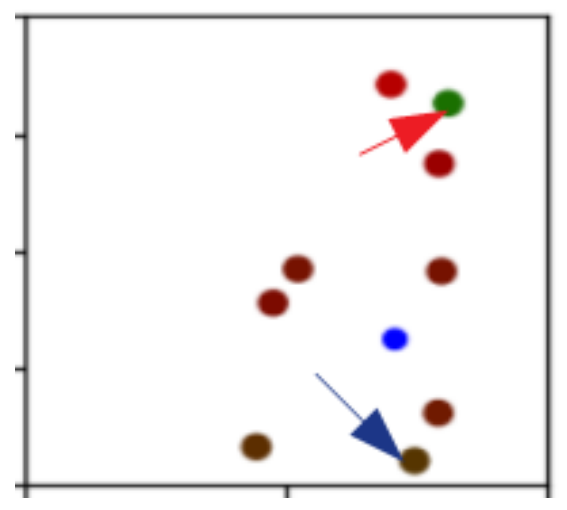

(d) PCM episodic memory events map

Fig. 4: After a stable period (blue arrow in (c) and (d)), time projection found a pleasant memory (yellow arrow in (a), red arrow in (c) and (d)) and the PCM (blue dot in (a) and (d)) moves towards its recorded location.

\section{CONCLUSION}

The original PCM imagination engine allows agents to project themselves in a different spatial setting representing possible future factual outcomes based on prior belief. Adding a basic episodic memory and the possibility for agents to project themselves explicitly in their past, shows behavioral changes that are consistent with essential properties of nostalgia. As the PCM continues to be developed and becomes more sophisticated, we expect other characteristics of natural psychological phenomena such as rumination to emerge and be represented in a more precise way. 


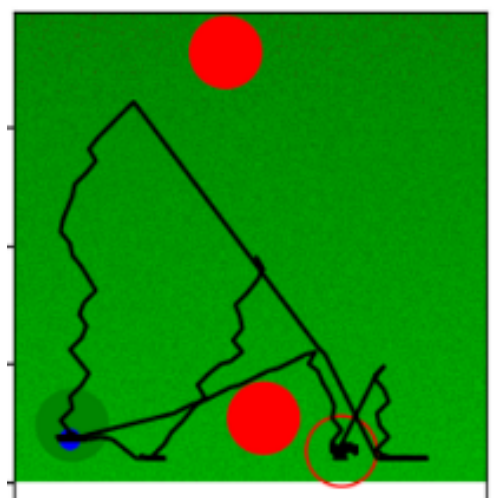

(a) World

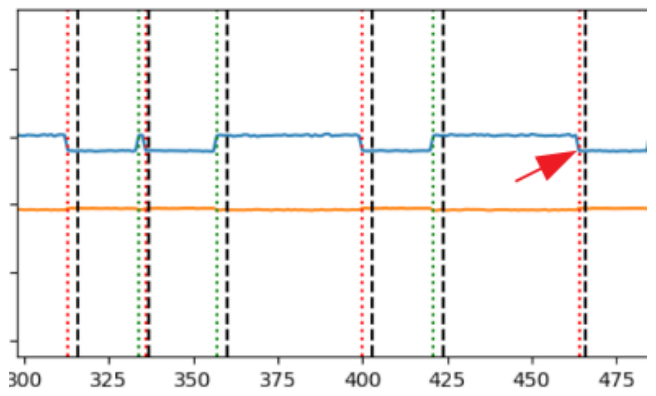

(c) Experienced FE timecourse

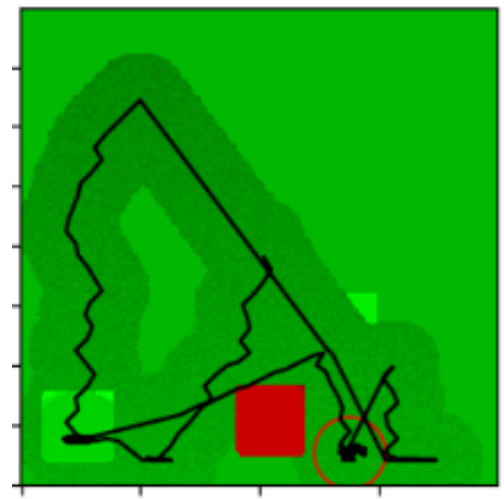

(b) PCM World Model

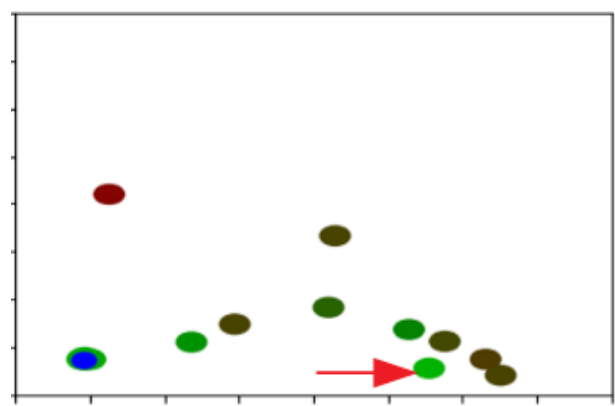

(d) PCM episodic memory events map

Fig. 5: Time projection found a pleasant memory (red arrow in (d)), but it does not exist anymore in the world model (b) (inside the red circle). The PCM mind-wanders but does not move towards the event's recorded location (red arrow in (d)). This decreases its FE (red arrow in (c).

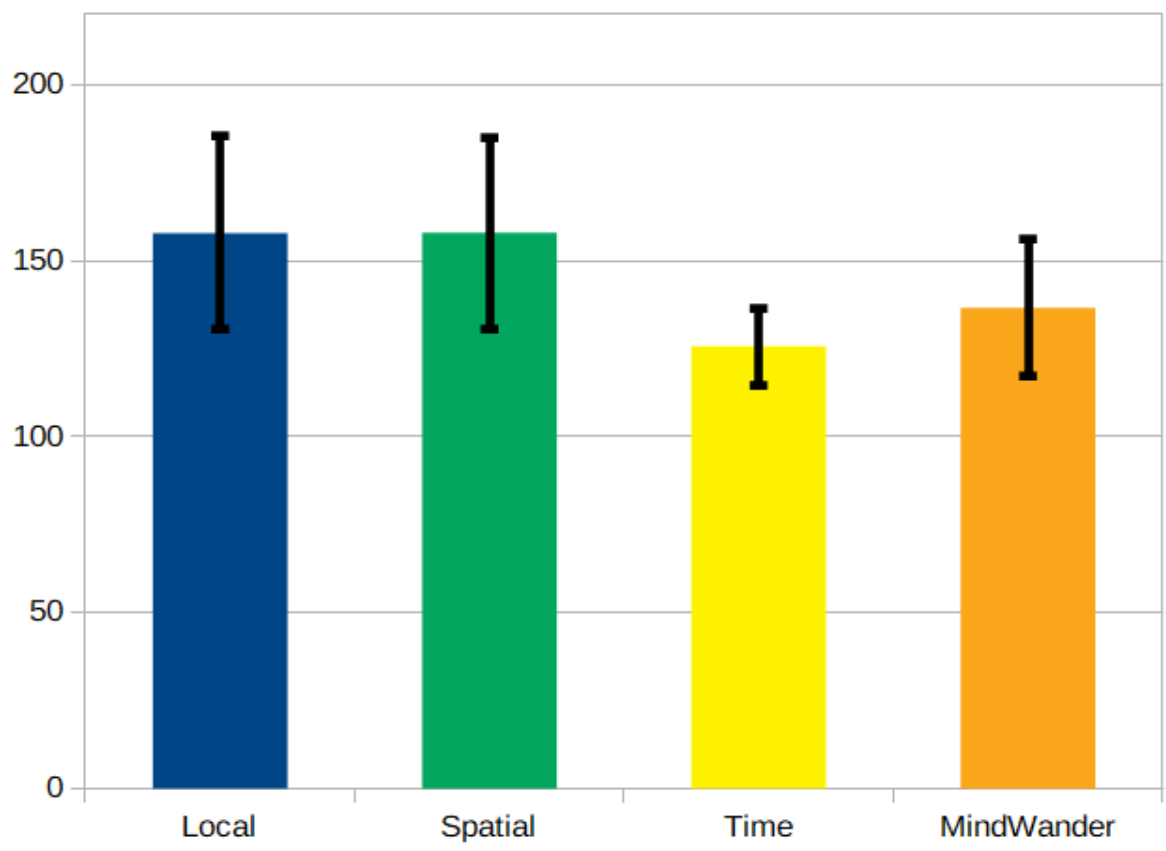

Fig. 6: FE values from 100 simulations. Mean FE after local optiomization, after spatial projection, after time projection and after mind-wandering. Error bars show the standard deviation of the FE values. 


\section{REFERENCES}

[1] Matthew Baldwin, Monica Biernat, and Mark J. Landau. Remembering the real me: Nostalgia offers a window to the intrinsic self. Journal of Personality and Social Psychology, 108(1):128, 20141027.

[2] Andrea Bocincova, Taylor Nelson, Jeffrey Johnson, and Clay Routledge. Experimentally induced nostalgia reduces the amplitude of the eventrelated negativity. Social Neuroscience, pages 1-4, February 2019.

[3] Susan Turk Charles, Mara Mather, and Laura L. Carstensen. Aging and emotional memory: The forgettable nature of negative images for older adults. Journal of Experimental Psychology: General, 132(2):310, 20030519.

[4] Daniel C. Dennett and Marcel Kinsbourne. Time and the observer: The where and when of consciousness in the brain. Behavioral and Brain Sciences, 15(2):183-201, 1992.

[5] Phoebe C Ellsworth and Klaus R Scherer. Appraisal processes in emotion. Handbook of affective sciences, 572:V595, 2003.

[6] Johnny RJ Fontaine, Klaus R Scherer, and Cristina Soriano. Components of Emotional Meaning: A Sourcebook. Oxford University Press, 2013.

[7] Karl Friston. The free-energy principle: A unified brain theory? Nature Reviews Neuroscience, 11(2):127-138, February 2010.

[8] Erica G. Hepper, Tim Wildschut, Constantine Sedikides, Timothy D. Ritchie, Yiu-Fai Yung, Nina Hansen, Georgios Abakoumkin, Gizem Arikan, Sylwia Z. Cisek, Didier B. Demassosso, Jochen E. Gebauer, J. P. Gerber, Roberto González, Takashi Kusumi, Girishwar Misra, Mihaela Rusu, Oisín Ryan, Elena Stephan, Ad J. J. Vingerhoets, and Xinyue Zhou. Pancultural nostalgia: Prototypical conceptions across cultures. Emotion, 14(4):733, 20140526.

[9] Mateus Joffily and Giorgio Coricelli. Emotional Valence and the FreeEnergy Principle. PLoS Computational Biology, 9(6):e1003094, June 2013.

[10] Agnes Moors, Phoebe C. Ellsworth, Klaus R. Scherer, and Nico H. Frijda. Appraisal Theories of Emotion: State of the Art and Future Development. Emotion Review, 5(2):119-124, April 2013.

[11] Chelsea A. Reid, Jeffrey D. Green, Tim Wildschut, and Constantine Sedikides. Scent-evoked nostalgia. Memory, 23(2):157-166, February 2015.

[12] David Rudrauf, Daniel Bennequin, Isabela Granic, Gregory Landini, Karl Friston, and Kenneth Williford. A mathematical model of embodied consciousness. Journal of Theoretical Biology, 428:106-131, September 2017.

[13] David Rudrauf and Martin Debbané. Building a Cybernetic Model of Psychopathology: Beyond the Metaphor. Psychological Inquiry, 29(3):156-164, July 2018.

[14] Klaus R Scherer, Angela Schorr, and Tom Johnstone. Appraisal Processes in Emotion: Theory, Methods, Research. Oxford University Press, 2001.

[15] Constantine Sedikides and Tim Wildschut. Finding meaning in nostalgia. Review of General Psychology, 22(1):48-61, March 2018.

[16] Wijnand A. P. van Tilburg, Martin Bruder, Tim Wildschut, Constantine Sedikides, and Anja S. Göritz. An appraisal profile of nostalgia. Emotion, 19(1):21, 20180305.

[17] Wijnand A. P. van Tilburg, Tim Wildschut, and Constantine Sedikides. Nostalgia's place among self-relevant emotions. Cognition and Emotion, 32(4):742-759, May 2018.

[18] Matthew Vess, Jamie Arndt, Clay Routledge, Constantine Sedikides, and Tim Wildschut. Nostalgia as a Resource for the Self. Self and Identity, 11(3):273-284, July 2012.

[19] Tim Wildschut, Constantine Sedikides, Jamie Arndt, and Clay Routledge. Nostalgia: Content, triggers, functions. Journal of Personality and Social Psychology, 91(5):975, 20061023.

[20] Kenneth Williford, Daniel Bennequin, Karl Friston, and David Rudrauf. The Projective Consciousness Model and Phenomenal Selfhood. Frontiers in Psychology, 9, December 2018. 